\title{
Examining the Relationships Among Concealment Tendencies, IIIness Attitudes, Belief in a Just World, and Cognitive Flexibility
}

\author{
Kyung Hwan Cha ${ }^{1}$, Hua Jin ${ }^{1}$, Jung Hee $\mathrm{Ha}^{2 *}$ and Juliet Jue ${ }^{3}$ \\ ${ }^{1}$ Department of Multicultural Education, Hanyang University, Seoul, South Korea, ${ }^{2}$ Graduate School of Counseling Psychology, \\ Hanyang University, Seoul, South Korea, ${ }^{3}$ Department of Art Therapy, Hanyang Cyber University, Seoul, South Korea
}

OPEN ACCESS

Edited by:

Andrea Greco,

University of Bergamo, Italy

Reviewed by:

Ronaldo Pilati,

University of Brasilia, Brazil

Robert Youmans,

Google, United States

*Correspondence:

Jung $\mathrm{Hee} \mathrm{Ha}$

hajung366@hanyang.ac.kr

Specialty section:

This article was submitted to

Cultural Psychology,

a section of the journal

Frontiers in Psychology

Received: 10 November 2020

Accepted: 07 June 2021

Published: 03 September 2021

Citation:

Cha KH, Jin H, Ha JH and

Jue $J$ (2021) Examining the

Relationships Among Concealment

Tendencies, IIIness Attitudes, Belief in a Just World, and Cognitive Flexibility.

Front. Psychol. 12:627739.

doi: 10.3389/fpsyg.2021.627739
The purpose of this study was to verify the relationships among concealment tendencies, illness attitudes, belief in a just world, and cognitive flexibility. The participants were 418 Korean and 400 Chinese adults. We conducted correlational analysis, structural equation modeling, and verification of mediating effects. We found that cognitive flexibility-control factor fully mediated the relationship between concealment tendencies and illness attitudes for Korean participants and partially mediated the relationship for Chinese participants. The relationship between concealment tendencies and cognitive flexibility-alternatives factor differed across participants' country of origin. For Chinese participants, cognitive flexibility-alternatives fully mediated the relationship between concealment tendencies and belief in a just world. These differences might stem from the countries' different social systems, values, and attitudes. Finally, we discuss this study's implications and limitations.

Keywords: concealment tendencies, illness attitudes, belief in a just world, cognitive flexibility, control, alternatives

\section{INTRODUCTION}

Asian cultures strongly value groups and communities, and Asian people are known to be highly sensitive to others' judgments. For example, Koreans feel more embarrassed when they make mistakes in front of others than while alone, and they try to hide these public mistakes (Lee and Kim, 2006). These cultural characteristics are common not only for Koreans but also for Chinese. In collectivist cultures, unity and harmony with others are of great importance (Wang, 2011). Collectivist cultures allow people to rely on and cooperate with each other; however, they may overemphasize interpersonal relationships, making people hyperconscious of what others think or appreciate. People in collectivist cultures fear public mistakes, change or hide their opinions based on others' evaluations, and strive to maintain perfect images of themselves (Wang, 2011). Thus, concealing mistakes or flaws could serve to protect one's perceived perfection.

Concealment tendencies are related to perfectionistic self-presentation, which is defined as the desire to appear perfect to others (Hewitt et al., 2003). People with such tendencies tend to show only their perfect qualities and hide imperfect behavior or language. Sub-factors of perfectionistic self-presentation include perfectionistic self-promotion (PSP), non-display of imperfection (NDP), and non-disclosure of imperfection (NDC). Concealment tendencies comprise the latter two categories. 
Concealment tendencies are associated with psychological and behavioral maladjustment (Kim and Seo, 2009; Hong and Hong, 2011; Kim and Hyun, 2012; Kim and Lee, 2013; Park and Yang, 2014). Previous studies have shown that the characteristics of perfectionism dominated by concealment tendencies include fear of rejection (Wu and Wei, 2008; Flett et al., 2014), negativity bias (Kim and Hyun, 2012), maladaptive emotions (Go and Hyeon, 2009), and selective disclosure of imperfection (Hewitt et al., 2003; Lee and Suh, 2010; Baek and Lee, 2013). In contrast, Li et al. (2006b) reported on the positive functions of concealment tendencies. The researchers argue that concealment tendencies give people a sense of psychological stability by promoting a positive self-image, avoiding the hatred of others, and preventing harm to the individual's reputation. Further, short-term self-concealment is advantageous for perfectionists to avoid the evaluation of others, so this can also be viewed as an adaptive behavior. Additionally, research has determined that adolescents' concealment of themselves from their parents may be beneficial for the development of emotional autonomy ( $\mathrm{Li}$ et al., 2006a).

Nonetheless, with the global spread of coronavirus disease (COVID-19) in 2020, individuals with strong concealment tendencies are more likely to experience anxiety and health concerns due to their personality traits. As pointed out by $\mathrm{Lu}$ et al. (2009), those with strong concealment tendencies also are more likely to develop stress-related illnesses. The spread of infectious disease augments people's health concerns. Kim et al. (2020) conducted a survey of 1,000 Koreans and found that, compared with natural disasters or non-contagious diseases, COVID-19 has induced higher levels of stress and anxiety. Similarly, Chinese citizens have also reported feeling high levels of anxiety and fear from the spread of COVID-19 (Li et al., 2020).

Additionally, the issue of quarantine beyond the individual level has become important. When their anxiety increases, people may lose faith in society or develop negative attitudes. Yang and Wang (2020) reported that the spread of COVID-19 has undermined college students' confidence in the government and has created tensions between the government and citizens, threatening stability. On the contrary, Li et al. (2020) surveyed citizens both within and outside Hubei, the first COVID-19 outbreak area, and reported that their satisfaction with the government's countermeasures was high (85.7 and 90.0\%, respectively). Kim et al. (2020) conducted a similar survey on metropolitan area residents in Korea and reported that $63.3 \%$ of study participants were satisfied with the government's countermeasures. One of the reasons why people evaluate the government's response differently might be that individuals have different levels of security or belief in their society and country. In this study, we examined whether Korean and Chinese citizens feel secure in their respective countries through belief in a just world.

Belief in a just world is defined as personal assumptions that the world is basically fair (Lerner, 1977). These beliefs provide psychological stability by recognizing the physical and social environment to which an individual belongs as orderly and stable (Lerner, 1977; Dalbert, 1999; Furnham, 2003). There may be unavoidable inequality in various areas (e.g., wealth, educational opportunities, and welfare), but responses to the unfairness vary from person to person (Nudelman and Shiloh, 2011). The belief that everything in the world happens fairly creates hope, trust, and confidence despite an uncertain future (Du et al., 2007; Zhen, 2013). Previous research has demonstrated that perceptions of unfairness cause psychological maladjustment, such as depression and anxiety, and cognitive flexibility mediates this process (Kim et al., 2012; Choi and Shin, 2018; Shin, 2018).

Cognitive flexibility refers to the ability to perceive that one can control difficult situations and produce a variety of alternatives to the problem at hand (Dennis and Vander Wal, 2010). It is the ability to change (Thurston and Runco, 1999), the spontaneity to adapt to situations, recognizing that there are new alternatives and choices an individual can make (Martin and Rubin, 1994; Heo, 2011), or personal ability to cope with unexpected changes (Gough, 1995). Thus, a measure of cognitive flexibility is the degree to which an individual challenges negative thinking and restructures the situations when faced with difficulties (Cho and Jo, 2017).

Cognitive flexibility is divided into two sub-factors: alternatives and control. While the former involves self-efficacy with respect to presenting alternative interpretations of and solutions to difficult situations, the latter is defined as the tendency to perceive the situation as controllable (Dennis and Vander Wal, 2010). According to Ellis (2001), rigid thinking, including irrational thoughts or self-defeating beliefs, causes cognitive distortion and increases anxiety. In contrast, cognitive flexibility promotes positive problem solving and lowers emotional distress, including anxiety (Johnco et al., 2013; Kim and You, 2017; Shin, 2018). Wang et al. (2019) found that cognitive flexibility (both dimensions) predicted belief in a just world. Heo (2011) reports that cognitive flexibility-control exhibited strong negative correlations with dysfunctional perfectionism, depression, and anxiety. The researcher also found that cognitive flexibility-control partially mediated the relationship between dysfunctional perfectionism and depression as well as the relationship between dysfunctional perfectionism and anxiety. Therefore, we assume that cognitive flexibility mediates the process of concealment tendencies affecting illness attitudes and belief in a just world.

Based on the preceding studies, we hypothesized that the relationships among concealment tendencies, illness attitudes, belief in a just world, and cognitive flexibility would be as they are presented in Figure 1. In an effort to determine the existence of cultural differences, we examined the relationships among the foregoing variables for both Korean and Chinese participants.

\section{MATERIALS AND METHODS}

\section{Participants and Procedures}

We surveyed a total of 818 adults in the Republic of Korea and China. In the Korean subsample, there were 145 men (34.7\%) and 273 women (65.3\%). Their age distribution was as follows: 106 in their 20s (25.4\%), 122 in their 30s (29.2\%), 94 in their $40 \mathrm{~s}(22.5 \%), 94$ in their 50s (22.5\%), and 2 in 


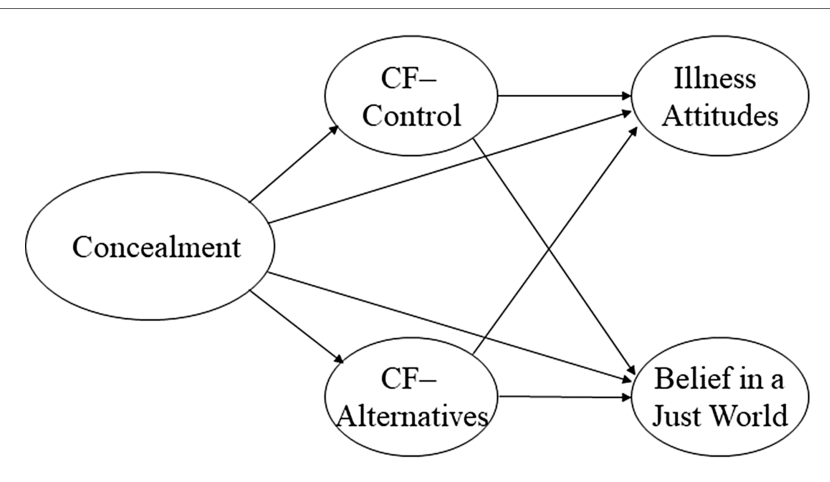

FIGURE 1 | Research Model.

their $60 \mathrm{~s}$ or older $(0.5 \%)$. One hundred and ninety-four were married $(46.4 \%), 212$ were single/divorced/widowed (50.7\%), and 12 specified "other" or provided no answer to the marital status item $(2.9 \%)$. With respect to past disease experiences, 105 participants $(25.1 \%)$ had diseases, $311(74.4 \%)$ had no serious disease, and two specified "other" or provided no answer to the item $(0.5 \%)$. Regarding current disease experiences, 71 participants (17.0\%) have diseases, while 347 $(83.0 \%)$ have no serious disease. As for educational background, $86.2 \%$ were college graduates, and $13.6 \%$ were high school graduates.

In the Chinese subsample, there were 181 men $(45.3 \%)$ and 219 women $(54.7 \%)$. Their age distribution was as follows: 215 in their 20 s $(53.6 \%), 75$ in their 30 s (18.8\%), 40 in their 40 s $(10.0 \%), 67$ in their 50s $(16.8 \%)$, and 3 in their 60 s and over $(0.8 \%)$. Two hundred and sixteen were married $(54.0 \%)$, 181 were single/divorced/widowed (45.2\%), and three specified "other" or provided no answer to this item $(0.8 \%)$. With respect to past disease experiences, 32 participants $(0.8 \%)$ had diseases, while 368 (92.0\%) had no serious disease. Regarding current disease experiences, 31 participants $(7.8 \%)$ have diseases, while $369(92.2 \%)$ have no serious disease. As for educational background, $78.7 \%$ were college graduates, and $15.5 \%$ were high school graduates.

The survey period lasted from April 2020 to May 2020. We used both paper and online surveys. After excluding incomplete paper surveys and online surveys completed too rapidly (less than $100 \mathrm{~s}$ ), 418 Korean questionnaires and 400 Chinese questionnaires remained for the final analysis.

\section{Measures}

\section{Perfectionistic Self-Presentation Scale}

Hewitt et al. (2003) developed the Perfectionistic SelfPresentation Scale (PSPS). We used the PSPS-Korean Version translated and modified by $\mathrm{Ha}$ (2011) to measure Korean participants' concealment tendencies. The scale comprises 19 items. In this study, we used 11 items from the scale representing two domains: (1) NDP and (2) NDC. Items were rated on a 7-point Likert scale ranging from 1 (not at all likely) to 7 (extremely likely). Higher total scores represent higher levels of perfectionistic self-presentation. Ha reported a Cronbach's $\alpha$ of 0.85 for the full scale. In this study, we found that
Cronbach's $\alpha$ to be 0.76 for all items, 0.67 for NDP, and 0.66 for NDC.

For Chinese participants, we used the PSPS-Chinese Version translated and validated by $\mathrm{Yu}$ (2007). This scale comprises 21 items, and we used 12 items from the scale representing the same two domains as above. Its rating scale and interpretation of total scores match those of the PSPS-Korean Version. Yu reported a Cronbach's $\alpha$ of 0.86 for the full scale. In this study, we found that Cronbach's $\alpha$ to be 0.82 for all items, 0.74 for NDP, and 0.77 for NDC.

\section{Cognitive Flexibility Inventory}

To measure cognitive flexibility, we used the Cognitive Flexibility Inventory (CFI) developed by Dennis and Vander Wal (2010). For Korean participants, we used the CFI-Korean Version translated and modified by Heo (2011). This inventory comprises 19 items representing two domains: control (eight items) and alternatives (11 items). The cognitive flexibility-control subscale measures the tendency to perceive difficult situations as controllable. For example, respondents answer statements, such as When I face a difficult situation, I feel like I will lose control; When I face a difficult situation, I get so stressed that I can't think of any way to deal with it.

On the other hand, the cognitive flexibility-alternatives subscale measures the ability to conceive various alternative explanations for life events and human behaviors and produce alternative solutions to difficult situations. For example, this scale presents statements, such as When faced with difficult situations, I consider various options before acting; I tend to view difficult situations from different points of view. These two subscales measure different constructs: The cognitive flexibility-alternative factor measures the consideration of various solutions. The cognitive flexibility-control subscale measures the sense of efficacy based on the belief in flexibility (Johnco et al., 2014).

Items were rated on a 5 -point Likert scale ranging from 1 (not at all likely) to 5 (extremely likely). Higher total scores represent higher levels of cognitive flexibility. Heo (2011) reported high internal consistency for the full scale $(\alpha=0.86)$. In this study, we found that Cronbach's $\alpha$ to be 0.90 for all items, 0.84 for Cognitive Flexibility-Control, and 0.90 for Cognitive Flexibility-Alternative.

For Chinese participants, we used the CFI-Chinese Version translated and validated by Wang et al. (2016). The scale comprises 20 items, including 8 items for control and 12 items for alternatives. The characteristics of the scale match those of the CFI-Korean Version. Wang et al. reported the Cronbach's $\alpha$ for the scale as 0.88 . We found that Cronbach's $\alpha$ to be 0.88 for all items, 0.79 for Cognitive Flexibility-Control, and 0.92 for Cognitive Flexibility-Alternative.

\section{Illness Attitudes Scale}

To measure illness attitudes, we used the Illness Attitudes Scale (IAS) developed by Kellner (1987). For Korean participants, we used the IAS-Korean Version translated and validated by Yi (2004). This scale consists of 27 items spanning eight factors: (1) fear of illness, (2) treatment experiences, (3) health habits, 
(4) effects of symptoms, (5) fear of disease, (6) disease conviction, (7) obsession with somatic sensations, and (8) fear of death. Items were rated on a 5-point Likert scale ranging from 1 (not at all likely) to 5 (extremely likely). Higher total scores reflect more negative illness attitudes. The scale demonstrated high internal consistency in Yi's study $(\alpha=0.86)$. We found Cronbach's $\alpha$ to be 0.84 for the total scale.

For Chinese participants, we used the IAS-Chinese Version produced by Luo et al. (2013). The scale comprises 20 items representing four domains: (1) fear of illness and death, (2) effects of symptoms, (3) behaviors of prevention and treatment of disease, and (4) doubts about one's own health. Its rating scale and interpretation of total scores match those of the IAS-Korean Version. Luo et al. reported the subscales' reliability coefficients as $0.82,0.82,0.74$, and 0.68 , respectively. We found Cronbach's $\alpha$ to be 0.80 for the total scale.

As described above, we used the IAS developed by Kellner (1987) in Korea and China; the scale used by the two countries is the same. Researchers validated the IAS in Korea and China and found results related to depression and anxiety in both countries (Kim, 2005; Yi, 2004; Luo et al., 2013). However, its sub-factors were different for each country, with eight factors for Korea and four factors for China. Thus, we randomly allocated items to three parcels, which were the same for the two countries' samples, and used them for analysis.

\section{Belief in a Just World Scale}

To measure belief in a just world, we employed the Belief in a Just World Scale (BJW) developed by Dalbert (1999). For Korean participants, we used the BJW-Korean Version translated and revised by Jung and Ahn (2017). It has two subscales: (1) BJW-self and (2) BJW-others. The scale consists of 13 items, each rated on a 6-point Likert scale ranging from 1 (not at all likely) to 6 (extremely likely). Higher total scores indicate stronger belief in a just world. Jung and Ahn reported high internal consistency for the full scale in their study ( $\alpha=0.88$ ). We found that Cronbach's $\alpha$ for all items was 0.85 . For sub-factors, Cronbach's $\alpha$ for personal belief was 0.78 , and Cronbach $\alpha$ for general belief was 0.78 .

For Chinese participants, we used the BJW-Chinese Version translated and revised by $\mathrm{Su}$ et al. (2012). The characteristics of the scale match those of the BJW-Korean Version. Su et al. reported high internal consistency for the full scale $(\alpha=0.89)$. In this study, we found that Cronbach's $\alpha$ to be 0.94 for all items, 0.93 for personal belief, and 0.88 for general belief sub-scale.

\section{Analysis Method}

We used AMOS 20 and IBM's SPSS Statistics 24.0 to test study hypotheses through structural equation modeling. We calculated the reliability for each measured variable in addition to calculating descriptive statistics and conducting correlational analysis. We conducted a confirmatory factor analysis to verify the validity of the measurement model and used a structural equation to verify the validity of the theoretical model and the influences of the variables. Finally, we used phantom variables to verify the statistical significance and relative size of the mediating effect.

\section{RESULTS}

\section{Correlations Among Variables}

First, we conducted item parceling as suggested by Russell et al. (1998). Then, we calculated means and standard deviations (see Table 1) and computed correlations among the measured variables in both subsamples (see Table 2).

\section{Verification of Measurement Models}

We conducted confirmatory factor analysis to verify that the measured variables accurately reflected the latent variables. In the Korean subsample, the results of the confirmatory factor analysis were as follows: $x^{2}(55, N=418)=177.62, p<0.001$, $\mathrm{TLI}=0.91$, CFI $=0.93$, and RMSEA $=0.07$ (0.06 0.09). As for the Chinese subsample, the confirmatory factor analysis produced the following results: $x^{2}(55, N=400)=221.29$, $p<0.001$, TLI $=0.91$, CFI $=0.93$, and RMSEA $=0.09$ $(0.08 \sim 0.10)$. Thus, we judged the measurement models in this study to be suitable.

\section{Verification of Structural Models}

In the Korean subsample, we compared the research model and the modified model to determine the one that most adequately represents the structural relationships among the variables (see Table 3). In the modified model, there is neither a path from concealment tendencies to illness attitudes nor a path from concealment tendencies to belief in a just world. We conducted a chi-square test for comparison because the research model and the modified model are mutually nested models. In order for the modified model to replace the research model, the chi-square value needed to be 3.84 or greater when the degrees of freedom of the difference equaled 1 and the significance level was set to 0.05 . The research model had a chi-square value of 177.62 , while the modified model's corresponding value was 180.48. When the degrees of freedom of the difference equaled 2 , the chi-square value was 2.86 , representing a significant change $(p<0.001)$. Therefore, due to its superior fit, we selected the modified model as the final model.

Figure 2 displays the final model's path coefficients. Concealment tendencies were negatively related to control $(\beta=-0.34, p<0.001)$. Control was negatively related to illness attitudes $(\beta=-0.52, p<0.001)$ and positively related to belief in a just world $(\beta=0.50, p<0.001)$.

In the modified model for the Chinese subsample, there is no path from concealment tendencies to belief in a just world, no path from control to belief in a just world, and no path from alternatives to illness attitudes. The chi-square value for the research model was 221.29, whereas that of the modified model was 227.45. When the degrees of freedom of the difference equaled 3 , the chi-square value was 6.15 , representing a significant change $(p<0.001)$. Therefore, we selected the modified model for this subsample, as well, due to its superior fit. 
TABLE 1 | Descriptive statistics for measurement variables.

\begin{tabular}{|c|c|c|c|c|c|}
\hline & Variable & Mean & SD & Skewness & Kurtosis \\
\hline \multirow{5}{*}{ Korean subsample $(N=418)$} & Concealment & 4.53 & 0.83 & -0.11 & -0.04 \\
\hline & Cognitive flexibility-control & 3.43 & 0.75 & -0.05 & -0.46 \\
\hline & Cognitive flexibility-alternatives & 3.92 & 0.62 & -0.36 & -0.11 \\
\hline & Illness attitudes & 2.67 & 0.49 & 0.02 & -0.48 \\
\hline & Belief in a just world & 3.60 & 0.73 & -0.19 & 0.59 \\
\hline \multirow{5}{*}{ Chinese subsample $(N=400)$} & Concealment & 4.39 & 0.97 & -0.20 & -0.13 \\
\hline & Cognitive flexibility-control & 3.25 & 0.57 & 0.37 & -0.03 \\
\hline & Cognitive flexibility-alternatives & 3.51 & 0.64 & -0.47 & 1.22 \\
\hline & Illness attitudes & 2.61 & 0.64 & 0.36 & -0.08 \\
\hline & Belief in a just world & 4.05 & 0.98 & -0.18 & -0.01 \\
\hline
\end{tabular}

TABLE 2 | Correlation coefficients for measurement variables.

\begin{tabular}{|c|c|c|c|c|c|c|}
\hline S. No. & Variables & 1 & 2 & 3 & 4 & 5 \\
\hline 1 & Concealment & - & $-0.27^{* * *}$ & $0.30^{* *}$ & $0.36^{* *}$ & $0.19^{* *}$ \\
\hline 2 & Cognitive flexibility-control & $-0.17^{* * *}$ & - & $0.19^{* *}$ & $-0.43^{* *}$ & 0.09 \\
\hline 3 & Cognitive flexibility-alternatives & 0.05 & $0.42^{* *}$ & - & $0.11^{*}$ & $0.39^{* *}$ \\
\hline 4 & Illness attitudes & $0.18^{* *}$ & $-0.27^{* *}$ & -0.08 & - & $0.12^{*}$ \\
\hline 5 & Belief in a just world & -0.09 & $0.28^{* *}$ & $0.22^{* *}$ & 0.00 & - \\
\hline
\end{tabular}

Correlations upper half: Chinese subsample; correlations lower half: Korean subsample. ${ }^{*} p<0.05 ;{ }^{* *} p<0.01$.

TABLE 3 | Fit of research model and of modified model.

\begin{tabular}{|c|c|c|c|c|c|c|}
\hline & Model & $\chi^{2}$ & $d f$ & TLI & CFI & RMSEA \\
\hline \multirow{2}{*}{ Korean subsample $(N=418)$} & Research model & $177.62^{* * *}$ & 55 & 0.91 & 0.93 & $0.07(0.06-0.09)$ \\
\hline & Modified model & $180.48^{* * * *}$ & 57 & 0.91 & 0.93 & $0.07(0.06-0.08)$ \\
\hline \multirow{2}{*}{ Chinese subsample $(N=400)$} & Research model & $221.29^{* * * *}$ & 55 & 0.91 & 0.93 & $0.09(0.08-0.10)$ \\
\hline & Modified model & $227.45^{* * * *}$ & 58 & 0.91 & 0.93 & $0.09(0.07-0.10)$ \\
\hline
\end{tabular}

${ }^{* * *} p<0.001$.

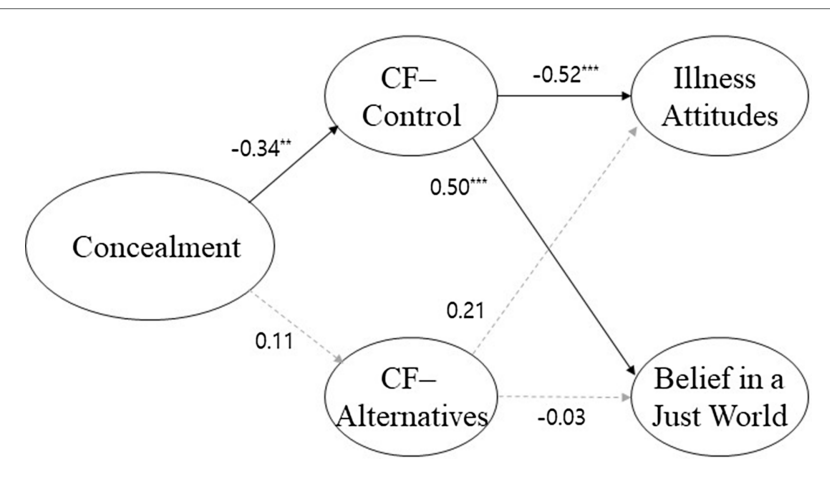

FIGURE 2 | Verified Structural Model for Korean Subsample.

Figure 3 displays the final model's path coefficients. Every path coefficient was significant. Concealment tendencies were negatively related to control $(\beta=-0.37, p<0.001)$ but were positively related to alternatives $(\beta=0.37, p<0.001)$ and to illness attitudes $(\beta=0.45, p<0.001)$. Control was negatively related to illness attitudes $(\beta=-0.34, p<0.001)$. Alternatives was positively related to belief in a just world $(\beta=0.42, p<0.001)$.

\section{Verification of Mediating Effects}

For Korean participants, control fully mediated the relationship between concealment tendencies and illness attitudes. Additionally, control fully mediated the relationship between concealment tendencies and belief in a just world. Thus, to confirm the magnitude of control's influence, we conducted a mediation effect verification using phantom variables (see Table 4). The mediating effect is considered significant if the confidence interval does not include zero. Control's mediating effect size in the relationship between concealment tendencies and illness attitudes was 0.03, $p<0.01$. The confidence interval's lower and upper limits were 0.01 and 0.05 , respectively, indicating a significant effect. Additionally, control's mediating effect size in the relationship between concealment tendencies and belief in a just world was -0.03 , $p<0.001$. The confidence interval's lower and upper limits were -0.07 and -0.01 , respectively, indicating a significant effect.

For Chinese participants, control partially mediated the relationship between concealment tendencies and illness attitudes. However, alternatives fully mediated the relationship between concealment tendencies and belief in a just world. We used the same analysis outlined above to confirm the magnitudes 


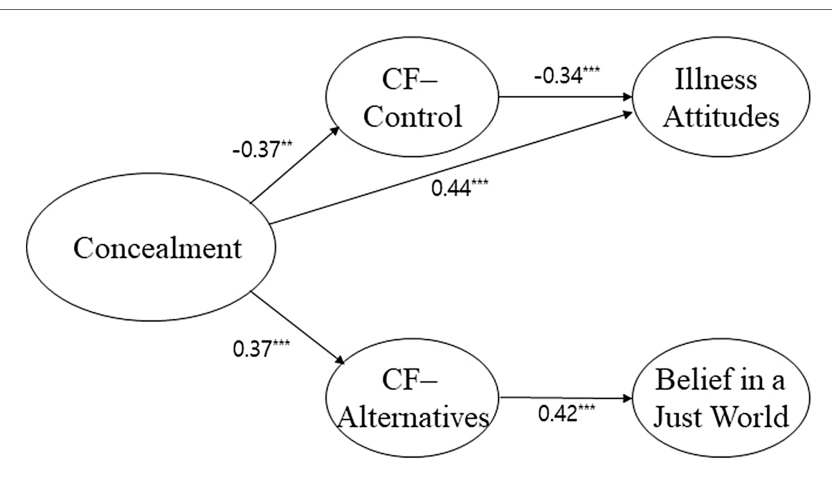

FIGURE 3 | Verified Structural Model for Chinese Subsample.

TABLE 4 | Verification results of mediating effect.

\begin{tabular}{llccc}
\hline & Path & Mediating & \multicolumn{2}{c}{$95 \%$ Cl } \\
\cline { 4 - 5 } & & effect & Lower & Upper \\
\hline Korean & $\mathrm{CT} \rightarrow$ Control $\rightarrow$ IA & $0.03^{* *}$ & 0.01 & 0.05 \\
$\begin{array}{l}\text { subsample } \\
(N=418)\end{array}$ & $\mathrm{CT} \rightarrow$ Control $\rightarrow$ BJW & $-0.03^{* *}$ & -0.07 & -0.01 \\
$\begin{array}{l}\text { Chinese } \\
\text { subsample }\end{array}$ & $\mathrm{CT} \rightarrow$ Control $\rightarrow$ IA & $0.07^{* * *}$ & 0.04 & 0.10 \\
$(N=400)$ & $\mathrm{CT} \rightarrow$ Alternatives $\rightarrow$ BJW & $0.12^{* * *}$ & 0.07 & 0.19 \\
\hline
\end{tabular}

CT, concealment tendencies; IA, illness attitudes; BJW, belief in a just world; and $\mathrm{Cl}$, confidence interval. ${ }^{* *} p<0.01 ;{ }^{* * *} p<0.001$.

of the respective influences of control and alternatives. We found that control's mediating effect size in the relationship between concealment tendencies and illness attitudes was $0.07, p<0.001$. The confidence interval's lower and upper limits were 0.04 and 0.10 , respectively. Although this is a significant result, we confirmed that control partially mediated the relationship because of its significant direct effect on illness attitudes ( $\beta=0.45, p<0.001)$. Alternatives' mediating effect size in the relationship between concealment tendencies and belief in a just world was $0.12, p<0.001$. The confidence interval's lower and upper limits were 0.07 and 0.19 , respectively; thus, this mediating effect was also significant.

\section{DISCUSSION}

We sought to examine the personally and socially maladaptive characteristics of concealment tendencies and to illuminate their process in a social atmosphere where anxiety caused by COVID-19 is prevalent. To this end, we examined both personal perceptions (illness attitudes) and social perceptions (belief in a just world) of those with strong concealment tendencies. Additionally, we examined the role of cognitive flexibility in the relationships among concealment tendencies, illness attitudes, and belief in a just world. Next, we discuss this study's results and implications.

Concealment tendencies were negatively correlated with cognitive flexibility-control in both the Korean and Chinese subsamples. This indicates that individuals demonstrating higher levels of concealment tend also to have lower levels of cognitive flexibility with respect to control. Individuals with strong concealment tendencies are mindful that their shortcomings may be revealed through words or actions (Winnicott, 1960; Schlenker and Leary, 1982; Hewitt et al., 2003). However, because it is impossible to completely conceal their imperfections, they might experience anxiety (Clark, 2005). Moreover, greater obsession with concealing imperfections may lower their cognitive flexibility, which could lead to psychological maladjustment (Heo, 2011). In other words, people with a strong tendency to fear evaluation may be less cognitively flexible because they cannot easily accept others' evaluations (Park and Hong, 2019). Thus, our results align with Shi and Qian's (1998) finding that those who are easily embarrassed avoid, deny, and distort reality by concealing their problems rather than solving them in difficult situations.

Cognitive flexibility-control was significantly negatively correlated with illness attitudes in both subsamples. Higher levels of the control aspect of cognitive flexibility are associated with less negative attitudes toward disease. Cognitive flexibility increases adaptability and enables appropriate responses in difficult situations, which directly affects anxiety reduction mechanisms, including illness attitudes. Low cognitive flexibility, in contrast, increases the likelihood of experiencing depression and anxiety (Kim and Seo, 2009; Heo, 2011; Kim and Lee, 2013; Lee and Yang, 2015).

In all other relationships among the factors, the differences between the two countries were prominent. One notable difference between the results from the Korean and Chinese subsamples was the relationship between concealment tendencies and cognitive flexibility-alternatives. For Chinese, concealment tendencies were significantly positively correlated with cognitive flexibility-alternatives. Cognitive flexibility involves the ability to replace maladaptive thoughts with more adaptive ones, and the alternatives aspect refers to the ability to switch one's cognitive framework in response to changing environmental stimuli (Wang et al., 2016). Therefore, we suggest that Chinese individuals, who often demonstrate strong concealment tendencies, are particularly adept at producing alternative explanations for events and solutions to problems.

Furthermore, for Chinese participants, cognitive flexibilityalternatives were significantly positively correlated with belief in a just world. That is, people with a stronger ability to devise alternative explanations and solutions are more likely to believe in a just world than their less cognitively flexible counterparts. This finding aligns with the results from Wang et al's (2019) study. The researchers found that cognitive flexibility influenced belief in a just world, with the alternatives aspect exerting greater influence than the control aspect.

Although belief in a just world was related to cognitive flexibility in both subsamples, the type of cognitive flexibility differed. For Koreans, the correlation with cognitive flexibilitycontrol was significant while that with alternatives was not significant. For Chinese, the correlation with cognitive flexibilitycontrol was not significant while that with alternatives was significant. We propose the two countries' different social systems and values as a plausible explanation for these different results. Before discussing this further, we elaborate on the cross-cultural differences in the results. 
We used structural equation modeling to verify the mediating effects of cognitive flexibility. We found that cognitive flexibilitycontrol fully mediated the relationship between concealment tendencies and illness attitudes for Koreans and partially mediated this relationship for Chinese. Previous research with Koreans has shown that those with high levels of perfectionistic self-presentation, including concealment tendencies, tend to perform maladaptive cognitive-emotional control behaviors (Kang, 2020). People with strong concealment tendencies typically fear being rejected by others in interpersonal relationships (Burns, 1980; Weisinger and Lobsenz, 1981; Hewitt et al., 2003), and they tend to avoid acknowledging their mistakes. They even hide their emotions, which consequently makes them feel less control over their situ ttion. Less control entails greater difficulty believing that the world is fair and more negative attitudes toward disease.

For Chinese participants (but not Korean participants), cognitive flexibility-alternatives fully mediated the relationship between concealment tendencies and belief in a just world. This supports a previous study's finding that belief in a just world helps people reduce anxiety by coping with threats and restructuring justice at a cognitive level (Zhou, 2013).

Based on these results, it was apparent that although Korea and China have commonalities as collectivist cultures, there are cultural and psychological differences in the details. Regarding similarities, both Korea and China showed that individuals' concealment tendencies were negatively related to the cognitive flexibility-control factor. They became negative about disease as their cognitive flexibility-control decreased. The cognitive flexibility-control factor relates to perceiving difficult situations as controllable (Dennis and Vander Wal, 2010). Both Korean and Chinese individuals in the collectivist culture are embarrassed when they misbehave in front of others and hide their behaviors (Lee and Kim, 2006; Wang, 2011). People willing to minimize their mistakes in public situations and hide their imperfect selves are more likely to become anxious and more sensitive to social pressure. This anxiety can eventually lead to having diseases related to stress (Lu et al., 2009). Therefore, concealment tendencies pursuing a perfect public image negatively influenced illness attitude in both subsamples.

Regarding the differences between the two countries' results, we propose that the differences are due to divergent social systems, attitudes, and values. China has maintained a socialist government for decades. In socialist countries, the interests of the community tend to be prioritized over the interests of individuals. Something judged as being beneficial to the community is considered the most valuable. Correspondingly, if an individual or state has concealed a fact, the reason for concealment might be for the benefit of the community, and concealment might have been a deliberate choice. If people recognize the world in which these choices are made as orderly and stable, they may believe more strongly in a just world. This view of the social community is also revealed in the formation of public opinion (Ren and Xie, 2018). In 2016, a Communist Party of China (CPC) press conference suggested that public opinion must maintain the CPC's principles and share the CPC's thoughts, politics, and action. All forms of media must embody the CPC's will, propagate its claims, defend its authority and unity, love and protect it, and advocate on its behalf (Ren and Xie, 2018). Chinese citizens generally accept the government-run media. One citizen states, "It is an inevitable process for the country to develop without losing its focus" (Kumendedalingnv1, 2013). Another frames the media situation thus: "It is not good to cause unnecessary social anxiety" (Dali858, 2010). Given these perceptions, we can partially understand why there are differences between countries in the relationships among concealment tendencies, alternatives, and belief in a just world.

Although concealment tendencies are associated with maladaptive traits in Korea, there are positive aspects in China. Because of the culture of the socialist system described above, personal concealment tendencies increase the cognitive flexibilityalternative factor, which positively affects the social attitude of belief in a just world. For example, Li et al. (2020) conducted a citizens' survey in the first coronavirus outbreak area. They found that more than $85.7 \%$ of citizens were satisfied with the government's countermeasures. Amid uncertainty or inequality, people tend to have a sense of stability by having hope and confidence (Zhen, 2013). For Chinese individuals, the stronger the concealment tendencies, the more they consider cognitive-alternative factors, reducing anxiety and protecting their positive self-images and belief in a just world. In summary, for Koreans, concealment tendencies are personal attitudes that could be maladaptive, whereas, for Chinese individuals, concealment tendencies have positive and negative effects.

This study's results have several implications. First, we confirmed the relationships among concealment tendencies, illness attitudes, and belief in a just world. Our verification of mediating effects underscores the fact that cognitive factors influence personal attitudes and social viewpoints. Second, this study determined that people from two Asian countries exhibit differences in their concealment tendencies. Although most previous studies have focused on the negative effects of concealment, we suggest its potentially positive effect for Chinese individuals of increasing cognitive flexibility with respect to alternatives and strengthening belief in a just world. Third, our study results could prove useful in providing psychotherapy to those with illness anxiety. If clients with strong concealment tendencies demonstrate serious negative attitudes toward disease and resentment and criticism toward the world, their psychotherapists may need to explore clients' cognitive flexibility. For Koreans, specifically, it would be more effective to address the control dimension instead of the alternatives dimension of cognitive flexibility. In contrast, for Chinese, it would be more effective to target the control dimension if treating individual attitudes (e.g., illness attitudes) and to target the alternatives dimension if treating social viewpoints (e.g., belief in a just world).

Our study also has several limitations. First, we collected data by sampling people from multiple regions of Korea and China, but generalizability may still be limited, as we did not sample from all regions. Future studies may benefit from expanded sampling procedures. Second, we utilized a self-report questionnaire, which reflects individual or situational factors when participants 
respond to the questionnaire. Furthermore, there is also the possibility of responding to the questionnaire based on social desirability. It is possible that participants' true characteristics have been distorted in this regard. Future research could use more objective measures that consider the cultural backgrounds and values in each country as well as take a multifaceted approach (e.g., qualitative research). Finally, we used cross-sectional data measured by a self-report questionnaire for all variables. Therefore, there will be a limit to find a clear causal relationship.

\section{DATA AVAILABILITY STATEMENT}

The raw data supporting the conclusions of this article will be made available by the authors, without undue reservation.

\section{REFERENCES}

Baek, S. H., and Lee, Y. S. (2013). The mediating effects of ambivalence over emotional expressiveness between perfectionistic self-presentation and social anxiety. Korean J. Couns. 14, 385-400. doi: 10.15703/kjc.14.1.201302.385

Burns, D. D. (1980). The perfectionist's script for self-defeat. Psychol. Today 14, 34-52.

Cho, S. Y., and Jo, H. I. (2017). The mediating effects of cognitive flexibility and gender differences: worry, rumination and stress response. Korean J. Couns. Psychother. 29, 809-842. doi: 10.23844/kjcp.2017.08.29.3.809

Choi, H. J., and Shin, H. J. (2018). The relationship between belief in a just world and anxiety: testing a moderated mediation model of cognitive flexibility and entrapment. Korean J. Soc. Person. Psychol. 32, 19-38. doi: 10.21193/ kjspp.2018.32.3.002

Clark, D. M. (2005). "A cognitive perspective on social phobia," in The Essential Handbook of Social Anxiety for Clinicians. eds. W. Crozier and L. E. Alden (New York: Wiley), 193-218.

Dalbert, C. (1999). The world is more just for me than generally: about the personal belief in a just world scale's validity. Soc. Justice Res. 12, 79-98. doi: 10.1023/A:1022091609047

Dali858 (2010). Under what circumstances does the country hide? Baiduzhidao. Available at: https://zhidao.baidu.com/question/168396365.html (Accessed July 20, 2010).

Dennis, J. P., and Vander Wal, J. S. (2010). The cognitive flexibility inventory: instrument development and estimates of reliability and validity. Cogn. Ther. Res. 34, 241-253. doi: 10.1007/s10608-009-9276-4

Du, J. Z., Zhu, Z. B., and Li, X. K. (2007). Development of just world scale for college students. Chin. J. Clin. Psych. 15, 239-241. doi: 10.3969/j. issn.1005-3611.2007.03.006

Ellis, A. (2001). Feeling Better, Getting Better, and Staying Better. CA: Impact Publishers.

Flett, G. L., Besser, A., and Hewitt, P. L. (2014). Perfectionism and interpersonal orientations in depression: an analysis of validation seeking and rejection sensitivity in a community sample of young adults. Psychiatry 77, 67-85. doi: $10.1521 /$ psyc.2014.77.1.67

Furnham, A. (2003). Belief in a just world: research progress over the past decade. Personal. Individ. Differ. 34, 795-817. doi: 10.1016/S0191-8869(02) 00072-7

Go, H. K., and Hyeon, M. H. (2009). The mediating effect of cognitive coping strategy on the relationship between perfectionism and anger expression. Korean J. Clin. Psychol. 28, 97-116. doi: 10.15842/kjcp.2009.28.1.006006

Gough, H. G. (1995). Guide Pratiqued Interpretation CPI. Practical Guide for Interpretation of the CPI. Paris: ECPA.

Ha, J. H. (2011). Development and validation study of perfectionistic selfpresentation scale-Korean version. Korean J. Couns. 12, 1121-1140.

Heo, S. Y. (2011). The role of cognitive flexibility in the relationship between perfectionism and psychological maladjustment. unpublished master's thesis. Seoul, South Korea: Seoul National University.

\section{ETHICS STATEMENT}

Ethical review and approval was not required for the study on human participants in accordance with the local legislation and institutional requirements. The patients/participants provided their written informed consent to participate in this study.

\section{AUTHOR CONTRIBUTIONS}

$\mathrm{JH}$ conceived and designed the analysis and contributed the data or analysis tools. $\mathrm{KC}$ and $\mathrm{HJ}$ collected the data. $\mathrm{KC}$ performed the analysis. $\mathrm{KC}, \mathrm{JH}$, and $\mathrm{JJ}$ wrote the paper. All authors read and approved the final manuscript.

Hewitt, P. L., Flett, G. L., Sherry, S. B., Habke, M., Parkin, M., Lam, R. W., et al. (2003). The interpersonal expression of perfection: perfectionistic selfpresentation and psychological distress. J. Pers. Soc. Psychol. 84, 1303-1325. doi: 10.1037/0022-3514.84.6.1303

Hong, K. H., and Hong, H. Y. (2011). The effects of fear of negative evaluation and cognitive emotional regulation on adolescents' social anxiety. Korean J. Youth Stud. 18, 291-319.

Johnco, C., Wuthrich, V., and Rapee, R. (2013). The role of cognitive flexibility in cognitive restructuring skill acquisition among older adults. J. Anxiety Disord. 27, 576-584. doi: 10.1016/j.janxdis.2012.10.004

Johnco, C., Wuthrich, V., and Rapee, R. (2014). The influence of cognitive flexibility on treatment outcome and cognitive restructuring skill acquisition during cognitive behavioural treatment for anxiety and depression in older adults: results of a pilot study. Behav. Res. Ther. 57, 55-64. doi: 10.1016/j. brat.2014.04.005

Jung, E. K., and Ahn, D. H. (2017). Validations of the belief in a just world scale for adolescents. J. Learner-Centered Curric. Instr. 17, 449-470. doi: 10.22251/jlcci.2017.17.16.449

Kang, M. J. (2020). Mediation of cognitive emotion regulation strategies between young adults' perfectionistic self-presentation and romantic relationship satisfaction. unpublished master's thesis. Seoul, South Korea: Hanyang University.

Kellner, R. (1987). A symptom questionnaire. J. Clin. Psychiatry 48, 268-274.

Kim, S. (2005). Uncertainty, illness attitude and state anxiety in kidney transplant patients. unpublished master's thesis. Seoul, South Korea: Ewha Woman's University.

Kim, S. H., and Hyun, M. H. (2012). The effect of perfectionism on facial expression interpretation and memory in evaluation situation. Korean $\mathrm{J}$. Psychol. Gen. 31, 27-44.

Kim, E. H., Kim, D. Y., and Kim, S. Y. (2012). The relationship between belief in a just world and Hwa-Byung symptoms: distributive and procedura justice beliefs. Korean J. Couns. 17, 25-45. doi: 10.15703/kjc.17.5.201610.25

Kim, S. J., and Lee, Y. S. (2013). The relationship between evaluative concerns perfectionism and social anxiety in college students: the mediating effects of fear of negative and positive valuation and cognitive emotional regulation. Korean J. Couns. 14, 1919-1938. doi: 10.15703/kjc.14.3.201306.1919

Kim, J. H., Oh, J. H., Kim, T. Y., Woo, J. G., Choi, H. J., Lee, D. K., et al. (2020). Corona 19 as a Gyeonggi-do province citizen views. Issue Diagnosis, 1, 1-25.

Kim, M. S., and Seo, Y. S. (2009). The relation between evaluative concern perfectionism and social anxiety: testing the mediating effects of fear of negative evaluation and self-presentational motivation. Korean J. Psychol. Gen. 28, 525-545.

Kim, J. H., and You, H. S. (2017). The mediating effects of cognitive flexibility social anxiety between gratitude and psychological well-being of high school students. J. Learner-Centered Curric. Instr. 17, 799-818. doi: 10.22251/ jlcci.2017.17.21.799

Kumendedalingnv1 (2013). Why does the country hide from the people. 360wenda. Available at: https://m.wenda.so.com/q/1377334416066792 (Accessed August 24, 2013). 
Lee, C. W., and Kim, H. C. (2006). An analysis of influences of "Chemyon" (social-face) sensitivity, social anxiety and self-esteem on uncertainty avoidance tendency. Korean J. Soc. Person. Psychol. 20, 17-30.

Lee, D. G., and Suh, H. N. (2010). The characteristics of high and low perfectionistic self-presentation in college students. Korean J. Couns. 11, 91-110. doi: 10.15703/kjc.11.1.201003.91

Lee, S. W., and Yang, N. M. (2015). The mediating effect of internalized shame and ambivalence over emotional expressiveness on the relationship evaluative concerns perfectionism and social anxiety in college students. Korean J. Couns. 16, 225-244. doi: 10.15703/kjc.16.4.201508.225

Lerner, M. J. (1977). The justice motive: some hypotheses as to its origins and forms. J. Pers. 45, 1-52. doi: 10.1111/j.1467-6494.1977.tb00591.x

Li, Y., Cai, T. S., Wang, X. L., and Yang, X. Z. (2006a). The correlation research of self-concealment level and mental health in new recruits. Chin. J. Behav. Med. Sci. 15, 1021-1022. doi: 10.3760/cma.j.issn.1674-6554.2006.11.029

Li, W. H., Liu, B., Liu, M., Zhao, X. H., and Chen, Q. S. (2020). Investigation and analysis of public cognition and prevention awareness of corona virus disease 2019 inside and outside Hubei province. J. Jinan Univ. 41, 186-193. doi: $10.11778 /$ j.jdxb.2020.02.014

Li, D. P., Yu, M. Z., Wang, C. K., Xie, X. J., Zhou, L. M., and Zhu, X. M. (2006b). Self-disclosure and self-concealment in adolescence and the relationship between them and subjective well-being. Psychol. Dev. Educ. 22, 83-90. doi: 10.3969/j.issn.1001-4918.2006.04.014

Lu, D., Li, L. M., and Xiu, F. X. (2009). Self-concealment of high school students and its relation with self-consistency and congruence. Chin. J. Clin. Psych. 17, 96-97.

Luo, D. Y., Zhang, Y. X., Chen, W. Z., Yu, E. Y., Hu, J., Cai, H., et al. (2013). "Psychological behavioral skills and interventions: a validity study on the Illness Attitudes Scale-Chinese Version," in Proceedings of the 1st Academic Conference of the Behavioral Medicine Branch of the Zhejiang Academy of Sciences, 46-52.

Martin, M. M., and Rubin, R. B. (1994). Development of a communication flexibility measure. South. J. Commun. 59, 171-178.

Nudelman, G., and Shiloh, S. (2011). Who deserves to be sick? An exploration of the relationships between belief in a just world, illness causal attributions and their fairness judgements. Psychol. Health Med. 16, 675-685. doi: $10.1080 / 13548506.2011 .569730$

Park, H. Y., and Hong, H. Y. (2019). The relationship between evaluative concerns perfectionism and performance anxiety of university students: the mediating effects of cognitive flexibility and self-compassion. Korean J. Couns. 20, 23-40.

Park, J. H., and Yang, M. J. (2014). The relation between socially prescribed perfectionism and social anxiety of college students: the mediator effects of social support and fear of negative evaluation. Korean J. Couns. Psychother. 26, 363-388.

Ren, Y. L., and Xie, L. (2018). Xi Jinping: the party's paperwork is very important. People's Daily Online-CPCnews. Available at: http://cpc.people.com.cn/xuexi/ n1/2018/1204/c385474-30440485.html (Accessed December 4, 2018).

Russell, D. W., Kahn, J. H., Spoth, R., and Altmaier, E. M. (1998). Analyzing data from experimental studies: a latent variable structural equation modeling approach. J. Couns. Psychol. 45, 18-29. doi: 10.1037/0022-0167.45.1.18

Schlenker, B. R., and Leary, M. R. (1982). Social anxiety and self-presentation: a conceptualization and model. Psychol. Bull. 92, 641-669. doi: 10.1037/0033-2909.92.3.641

Shi, C. S., and Qian, M. Y. (1998). Attribution styles and coping traits among shame prone persons. Chin. Ment. Health J. 12, 193-195.
Shin, H. J. (2018). Beliefs in a just world and depression: mediating effects of optimism and cognitive flexibility. J. Learner-Centered Curric. Instr. 18, 413-437. doi: 10.22251/jlcci.2018.18.5.413

Su, Z. Q., Zhang, D. J., and Wang, X. Q. (2012). Revising of belief in a just world scale and its reliability and validity in college students. Chin. J. Behav. Med. Brain Sci. 21, 561-563. doi: 10.3760/cma.j.issn.1674-6554.2012.06.026

Thurston, B. J., and Runco, M. A. (1999). Flexibility. Encycl. Creativity 1, 729-732.

Wang, L. (2011). Talk about the "face" of the Chinese. unpublished master's thesis. Heilongkiang, China: Heilongjiang University.

Wang, Z. X., Wang, X. X., and Xing, M. D. (2019). The effects of cognitive flexibility and contingencies of self-worth (CSW) on the belief in a just world among Chinese medical college students. China Higher Med. Educ. 7, 5-6. doi: 10.3969/j.issn.1002-1701.2019.07.003

Wang, Y., Yang, Y., Xiao, W. T., and Su, Q. (2016). Validity and reliability of the Chinese version of the cognitive flexibility inventory in college students. Chin. Ment. Health J. 30, 58-63. doi: 10.3969/j.issn.1000-6729.2016. 01.012

Weisinger, H., and Lobsenz, N. (1981). Nobody's Perfect. New York: Warner.

Winnicott, D. W. (ed.) (1960). "Ego distortion in terms of true and false self," in Maturational Process and the Facilitating Environment: Studies in the Theory of Emotional Development (New York, NY: International University Press), 140-152.

Wu, T. F., and Wei, M. (2008). Perfectionism and negative mood: the mediating roles of validation from others versus self. J. Couns. Psychol. 55:276. doi: 10.1037/0022-0167.55.2.276

Yang, Q. Q., and Wang, S. G. (2020). Thoughts on the political work of college students during the coronavirus outbreak. J. High. Educ. 11, 50-53.

Yi, I. H. (2004). Factor structure of the illness attitudes scale (IAS) in a Korean college sample. Korean J. Health Psychol. 9, 203-218.

$\mathrm{Yu}, \mathrm{H}$. R. (2007). A study on the relationship between perfectionistic selfpresentation, self-handicapping scale, and self-esteem scale. master's thesis, Jinan University. Wangfang data.

Zhen, W. H. (2013). An introduction to research on the theory of belief in the just world. J. Changchun Educ. Inst. 29, 14-15. doi: 10.3969/j. issn.1671-6531.2013.05.009

Zhou, C. Y. (2013). Individual and social functions of belief in a just world for different social classes. doctoral dissertation, Huazhong Normal University. Wangfang data.

Conflict of Interest: The authors declare that the research was conducted in the absence of any commercial or financial relationships that could be construed as a potential conflict of interest.

Publisher's Note: All claims expressed in this article are solely those of the authors and do not necessarily represent those of their affiliated organizations, or those of the publisher, the editors and the reviewers. Any product that may be evaluated in this article, or claim that may be made by its manufacturer, is not guaranteed or endorsed by the publisher.

Copyright (c) 2021 Cha, Jin, Ha and Jue. This is an open-access article distributed under the terms of the Creative Commons Attribution License (CC BY). The use, distribution or reproduction in other forums is permitted, provided the original author(s) and the copyright owner(s) are credited and that the original publication in this journal is cited, in accordance with accepted academic practice. No use, distribution or reproduction is permitted which does not comply with these terms. 